\title{
Carnets
}

Revue électronique d'études françaises de l'APEF

Première Série - 1 Numéro Spécial | 2009

Cultures littéraires : nouvelles performances \& développement

\section{La littérature, pour quoi faire? (brèves réflexions au pas de course)}

Franc Schuerewegen

\section{CpenEdition}

Journals

Édition électronique

URL : http://journals.openedition.org/carnets/3402

DOI : $10.4000 /$ carnets.3402

ISSN : 1646-7698

Éditeur

APEF

Édition imprimée

Date de publication : 1 juin 2009

Pagination : 17-22

Référence électronique

Franc Schuerewegen, «La littérature, pour quoi faire? (brèves réflexions au pas de course) », Carnets

[En ligne], Première Série - 1 Numéro Spécial | 2009, mis en ligne le 16 juin 2018, consulté le 03 mai 2019. URL : http://journals.openedition.org/carnets/3402 ; DOI : 10.4000/carnets.3402

\section{(c) (i) (3)}

Carnets est mis à disposition selon les termes de la licence Creative Commons - Atribution - Pas d'utilisation commerciale 4.0 International. 


\title{
LA LITTÉRATURE, POUR QUOI FAIRE ? \\ (brèves réflexions au pas de course)
}

FRANC SCHUEREWEGEN

U. Anvers /U. Nimègue

franc.schuerewegen@ua.ac.be

\begin{abstract}
Résumé
Antoine Compagnon, dans sa leçon inaugurale de 2007, défend la littérature et les recherches littéraires dans un cadre essentiellement moralisant et « ruskinien ». Yves Citton, quant à lui, plaide au même moment pour une lecture "actualisante " et "affabulante ». J'explique dans cet article pourquoi, en fin de compte, je préfère la méthode Citton à la méthode Compagnon.
\end{abstract}

\section{Abstract}

In his inaugural lecture (2006), Antoine Companon defends literature and literary studies in a moral and « ruskinian » manner. At the same moment, Yves Citton builds a reading model in which literary reading is defined as « actualisation » and " affabulation ». I try to explain in this article why I prefer, given the actual crisis of literary studies, the Citton method.

Mots-clés: Lecture littéraire, Textes possibles, Affabulation, Actualisation, Compagnon, Citton Keywords: Literary reading, Possible texts, Affabulation, Actualization, Compagnon, Citton 
... il s'agit de défendre la littérature et les études littéraires contre leurs ennemis, ce n'est pas facile. Je m'étonne à vrai dire que ce grand chercheur et immense proustien qu'est Antoine Compagnon ait pris le parti, dans sa leçon inaugurale de 2006 (j'ai repris son titre, je suis un peu faux derche, je l'avoue), de plaider la cause de la lecture littéraire, et de ceux qui en sont les spécialistes, à l'aide d'une série d'arguments dont je ne me rappelle que trop bien que Proust a démontré leur fragilité dans ses commentaires sur Ruskin : « J'ai essayé de montrer dans les notes dont j'ai accompagné ce volume que la lecture ne saurait être ainsi assimilée à une conversation, fût-ce avec le plus sage des hommes » («Journées de lecture », Pastiches et mélanges). Je trouve curieux que le professeur du Collège de France, homme informé, proustien éminent, choisisse dans son discours une position « ruskinienne », non «proustienne ». La lecture littéraire est une « conversation », un entretien avec les grands esprits des temps passés ${ }^{1}$. Le proustien a oublié Proust. Je m'explique mal cela... Une leçon inaugurale est aussi un exercice mondain. Le mondain aurait-il évincé de sa place le chercheur le 30 novembre 2006 ? C'est possible...

Nous reprenons donc, cette fois sans Antoine Compagnon. La littérature, pour quoi faire ? bis.

L'ouvrage d'Yves Citton a paru la même année que la leçon inaugurale d'Antoine Compagnon, il porte sur les mêmes questions, ou presque. Mais quelle différence de ton, et

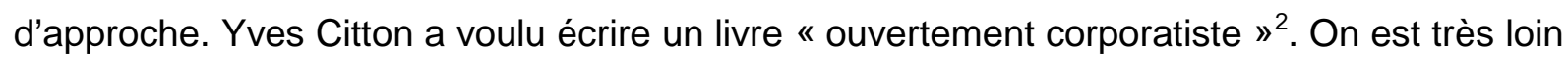
du ton feutré et conciliateur du maître de la rue des Ecoles, j'aime ce contraste, eh oui.

Pourtant, je ne suis pas d'accord avec tout. Yves Citton a un peu trop souvent tendance en ce qui me concerne - il a ce point en commun avec Antoine Compagnon -, à identifier - ou à réduire - l'œuvre littéraire au récit de fiction, c'est-à-dire grosso modo au roman. On part donc de l'idée que fiction et littérature sont à peu près la même chose, ce qui fait surgir sous la plume d'Yves Citton, pourtant si convaincant dans le rôle de pit-bull universitaire, une série de prises de position qu'on retrouve aussi dans l'ouvrage de l'homme du monde qu'est Antoine Compagnon : la littérature a une vertu édifiante, en la lisant, on part à la découverte « d'autres univers », on va vers l'autre ; bref : on connaît la musique et c'est toujours la même.

\footnotetext{
1 «La lecture littéraire », écrit Antoine Compagnon, «permet d'accéder à une expérience sensible et à une connaissance morale qu'il serait difficile, voire impossible, d'acquérir dans les traités des philosophes », p. 62 et : "La littérature doit donc être lue et étudiée parce qu'elle offre un moyen [...] de préserver et de transmettre l'expérience des autres », p. 63. La littérature, pour quoi faire ?, Collège de France/Fayard, 2007.

${ }^{2}$ Lire, interpréter, actualiser. Pourquoi les études littéraires?, Editions d'Amsterdam, 2007, p. 24.
} 
II est vrai qu'Yves Citton réinscrit l'argument « ruskinien » (j'appellerai ainsi tout défense de la culture littéraire dans un but essentiellement moralisant et paternaliste) dans le cadre d'une argumentation rigoureuse et puissante. Ce chercheur littéraire est aussi un philosophe et un spécialiste de Spinoza, cela se remarque, entre autres, au vocabulaire utilisé. Yves Citton n'a pas, lui, un style « feutré »:

Les fictions et leurs interprétations littéraires apparaîtront comme un espace unique de négociation de croyances, où peut se construire une culture proprement col-lective réfléchissant ensemble à ses lectures - qui déjoue à la fois les dangers d'une croyance rigidement bloquée (intégriste, fondamentaliste) et ceux d'une croyance excessivement labile (désengagée, désolidarisée, « opportuniste »). (Citton, 2007: 28)

Ou encore :

La fiction littéraire offre au lecteur l'occasion d'une délocalisation qui relève du mode utopique en ce que a) elle constitue un exercice mental sur les possibles latéraux à la réalité, et en ce que b) elle permet au lecteur d'expérimenter des réagencements affectifs capables de frayer de nouveaux possibles, en une époque où l'économie des affects devient le terrain de lutte central de nos développements sociétaux. (" thèse 34 », idem, p. 303)

J'objecterai pourtant à mon collègue de Grenoble que toute littérature n'est pas fictionnelle (que faire de Montaigne par exemple ? ou de certaines pages de Rousseau, ou de Proust ?) et que, en identifiant un peu trop rapidement littérature et fiction, Yves Citton attribue à la lecture littéraire un effet « thérapeutique » qu'on retrouve sans doute aussi - si on accepte que la lecture puisse être une thérapie -, du côté du cinéma, de la télévision, des jeux vidéo. La playstation (je parle en connaissance de cause, j'ai des enfants) propose à sa façon au joueur jeune « un exercice mental sur les possibles latéraux à la réalité » (Citton, 2007 : 305). A partir de là, je m'interroge. Si l'effet bénéfique que produit la lecture littéraire sur l'esprit du lecteur est le même que celui que procure une séance de playstation, on pourra tout aussi bien arrêter de lire et ne plus jouer qu'à la playstation (mes enfants ne demandent pas mieux). Je doute que ce soit exactement ce qu'Yves Citton cherche à nous dire ici.

Heureusement, le même auteur a d'autres flèches à son arc. Les meilleures pages de son livre sont celles où il décrit la lecture littéraire comme une exploration des « possibles » du texte et de la fiction. Je me permets de m'attarder un instant sur la question des «possibles », elle est cruciale comme on va voir.

Pour Yves Citton, les «possibles » littéraires sont nécessairement - puisque la littérature est faite de mots - des possibles verbaux, c'est-à-dire qu'ils sont textuels et non 
seulement situationnels. II y a là à coup sûr une différence avec la playstation, et le cinéma, et la fiction télévisuelle. "Expérimenter des possibles ", en littérature, ne revient pas seulement en effet à rêvasser ou à fantasmer sur une série de contenus - si j'étais Madame Bovary, si j'étais un Sims... -, la rêverie, si ce terme peut être utilisé, a ici une dimension proprement technique et - osons dire le mot - rhétorique : si j'étais moi-même écrivain, si j'avais à écrire l'histoire, ou le texte, que je suis en train de lire... Le lecteur littéraire certes s'imagine autre - comme le fait aussi le spectateur de cinéma, ou le jeune qui joue à la playstation - mais tout en s'imaginant autre, il se rend compte que les mots du texte auraient eux-mêmes pu être autres. En somme, les deux niveaux : la virtualité des situations, des contenus, et celle des mots, du support, vont nécessairement de pair.

II me semble que c'est la coïncidence des deux niveaux qu'Yves Citton choisit d'appeler dans son livre la «lecture actualisante». II entend par lecture actualisante un " usage » du texte littéraire où le lecteur ne se fait aucun scrupule de récupérer ce qu'il est en train de lire pour son horizon de vie propre, même - et peut-être surtout - si cet horizon n'a strictement rien à voir avec les choses évoquées dans le texte. Or la récupération est verbale, elle nécessite une expression, elle est une affaire de mots. Je cite Yves Citton :

Je lis le Discours de la servitude volontaire d'Etienne de la Boétie, et je " vois » dans le texte de cet écrivain de la Renaissance - comme je vois le pigeon assis sur le balcon d'en face - la description précise de nos divertissements télévisés du début du XXI siècle. (Citton, $2007: 26$ )

La lecture actualisante est un télescopage ; le lecteur, en quelque sorte, voit double, il est dans plusieurs contextes à la fois. Yves Citton ajoute que si «ce genre de pratique interprétative fait habituellement l'objet d'une sanction sans appel lors d'un examen ", et pour cause : l'examinateur condamnera «l'anachronisme », on peut aussi le « valoriser » et le « faire fructifier » car ce mode de lecture, pour blâmable qu'il puisse paraître -, mais qu'il n'est pas vraiment, selon Yves Citton -, « nourrit la pratique même de la littérature » (p. 27).

A vrai dire, la lecture actualisante est un premier élément qu'il a fallu relever pour décrire efficacement l'exploration des « possibles » tel que la conçoit Yves Citton; un second élément est nécessaire, j'y arrive maintenant: Yves Citton choisit de l'appeler la «lecture affabulante ». Qu'est-ce qu'une lecture « affabulante »?

Yves Citton emprunte le terme d'«affabulation » à Marc Escola, auteur d'un livre innovant sur les Fables de La Fontaine. Dans ce livre, on apprend que toute interprétation, quand l'objet de l'interprétation est un texte littéraire, est en vérité une affabulation, c'est-àdire qu'un texte que l'on lit, et auquel on cherche à donner un sens, est toujours en même temps un texte que l'on réécrit, ou que l'on réinvente.

Marc Escola écrit notamment : 
Notre usage du mot fable renvoie au modèle établi pour traiter la mythologie. Au centre de celui-ci, un énoncé qui fait l'objet d'une croyance et engage ceux qui la partagent ou la favorisent; cette réception et éventuellement cette production deviennent, dans un deuxième temps, l'objet d'une critique qui dénie à l'énoncé toute pertinence et traite la confiance qu'il suscite de folie ou de superstition. Par fable, on désigne le dispositif herméneutique qui articule autour d'un même énoncé deux interprétations divergentes, qui se succèdent ou entrent en concurrence. ${ }^{3}$

Je suis ici obligé d'ajouter que Marc Escola emprunte l'essentiel de ses idées à celui qui est en France le grand pionnier de la théorie des «textes possibles », je veux parler de Michel Charles, directeur de la revue Poétique, auteur de notamment Introduction à l'étude des textes, livre dont j'ai parlé ailleurs ${ }^{4}$. Je n'ai pas le temps de rendre compte en outre dans ces pages de la pensée de Michel Charles, qui est sinueuse et complexe. Que l'on sache qu'Yves Citton résume l'essentiel quand il écrit que, pour Michel Charles, «le donné textuel est par essence multiple » et "qu'il est absurde de parler de cohérence interne d'un donné textuel objectif - cohérence que l'interprète serait contraint par le texte lui-même de reconnaître - puisque c'est seulement à partir d'un certain modèle projeté sur le donné textuel qu'on peut parler de cohérence, de clôture, d'unité » (p. 87).

II y aurait ici lieu de s'interroger sur l'usage du terme d'interprétation dans le cadre d'une théorie des « textes possibles »; la lecture affabulante à proprement parler n'interprète pas, elle reformule et réinvente, elle ne relève pas d'une herméneutique, elle est rhétorique. Mais restons dans le sujet et revenons à Yves Citton et à la sorte de lien de solidarité qu'il aperçoit entre « interprétation », conçue comme réinvention et réécriture, et « affabulation » au sens escolien et charliste :

Cela décrit assez précisément le travail d'actualisation dont j'essaie d'expliquer et de légitimer la pratique au cours des différents chapitres de ce livre: les lectures actualisantes, au même titre que les lectures affabulantes, constituent la poursuite dans le présent d'un entretien commencé dans le passé, dont les variations et les adaptations possibles demandent encore à être explorées, à la fois pour éclairer l'avenir des suggestions esquissées hier, et pour se déniaiser des illusions dont on était victime jadis. (Citton, 2007 : 206)

Je comprends par là qu'explorer des « possibles », en littérature, c'est construire des petites «fables» locales et variables permettant au lecteur de faire en sorte qu'un texte

\footnotetext{
${ }^{3}$ Lupus in fabula. Six façons d'affabuler La Fontaine, Presses de l'Université de Vincennes, 2003, p. 45.

${ }^{4}$ Seuil, 1995. Je me permets de renvoyer à mon étude "Le critique ironiste ". Il y a une autre étude à faire sur Michel Charles et le philosophe pragmaticien américain (que Michel Charles n'a pas lu), Richard Rorty. Je l'écrirai un jour.
} 
d'autrefois ou venu d'ailleurs puisse recevoir une cohérence et une pertinence dans l'endroit où il se trouve. Je lis La Boétie et je pense à Thierry Ardisson ou à Claire Chazal...

Ici donc, nous disposons enfin d'un argument un peu solide - car force est d'admettre que la leçon inaugurale dont j'ai repris le titre ne nous a pas vraiment permis d'avancer pour défendre la recherche littéraire dans sa spécificité : l'analyse littéraire nous éclaire sur les conditions épistémologiques de toute analyse intellectuelle, quel que soit son objet. Par rapport à d'autres disciplines, la littérature peut fonctionner comme un modèle épistémologique. Je cite ici encore Yves Citton :

Cette modestie à l'égard des prétentions habituelles de la " parole vraie » se retourne toutefois aussitôt en revendication de supériorité : alors que toutes les autres disciplines ont une tendance inhérente à développer des dogmatismes, en ce qu'elles nourrissent une prétention (implicite) à décrire adéquatement une réalité extérieure à elles-mêmes, seules les études littéraires sont en mesure de se présenter ouvertement - et donc lucidement - pour ce qu'elles sont (et pour ce que sont en réalité tous les produits du savoir humain - : des affabulations, définies ici comme des constructions imaginaires tendant à se faire passer pour réelles. (Citton, $2007: 207$ )

Je paraphrase et conclus. Pourquoi la littérature est-elle utile ? Pour contribuer à la moralité publique ainsi que nous l'explique Antoine Compagnon ? Pas si sûr... Mieux vaut répondre - car c'est aussi pousser les choses beaucoup plus loin - que les départements de lettres sont absolument indispensables dans nos Universités - ce serait une formidable bêtise que de vouloir les supprimer - parce que nous sommes la seule discipline dans la panoplie académique qui parvienne effectivement, comme l'écrit Yves Citton, à « s'afficher dans sa fragilité constitutive ». Autrement dit, puisque, dès lors qu'il s'intéresse aux «possibles » d'une œuvre littéraire, l'interprète n'a d'autre choix que de se comporter en “ actualisateur », c'est-à-dire en "affabulateur », si l'on préfère, en inventeur et en producteur de son objet, il parvient ainsi à expliciter et à démasquer ce qui demeure le plus souvent implicite dans d'autres modes d'analyse intellectuelle et scientifique, à savoir que pour qu'on puisse disposer d'un objet à analyser, il faut d'abord le construire ; or le littéraire sait en outre, et ce n'est pas la partie la moins importante de son savoir, que l' invention à laquelle il procède n'est jamais définitive, et qu'elle est donc toujours à recommencer.

Quelque chose dans les pratiques d'analyse littéraire, conclut Yves Citton, s'approche « de la forme supérieure de la sagesse humaine »(Citton, $2007: 208)$.

L'homme a raison, ma foi.

Je n'ajoute pas : amen (j'en ai envie pourtant). 